\title{
A case of Rosai Dorfman disease
}

Priya Amaraweera ${ }^{1}$, HAS Perera ${ }^{2}$

${ }^{1}$ Registrar in Histopathology, ${ }^{2}$ Consultant Histopathologist, Karapitiya Teaching Hospital, Galle.

\section{Introduction}

Rosai Dorfman disease (RDD), or Sinus Histiocytosis with massive lymphadenopathy, is an idiopathic, usually self limiting, proliferative histiocytic disorder affecting lymph nodes [1]. Since this initial description several cases with extra nodal involvement have also been described. However Nodal RDD still remains the commonest mode of presentation [2].

\section{Case report}

A 32 year old female presented with gradually increasing bilateral neck swelling of several months duration. A cervical lymph node biopsy revealed granulomatous lymphadenitis with features favouring an atypical mycobacterial infection. The Mantoux test was negative and the ESR was $90 \mathrm{~mm}$. Other haematological investigations were within normal limits. The patient was commenced on anti TB therapy but there was no improvement.

Vol 11: No. 1, September 2006
CT scan of the neck showed findings in keeping with nodal lymphoma. Bone marrow aspiration and trephine biopsy however, were negative for lymphoma. The patient was referred to the Oncological Surgeon and bilateral cervical block dissection was performed.

On gross examination, the multiple enlarged lymph nodes were matted together and the cut surface was pale grey, firm and rubbery (Fig 1).

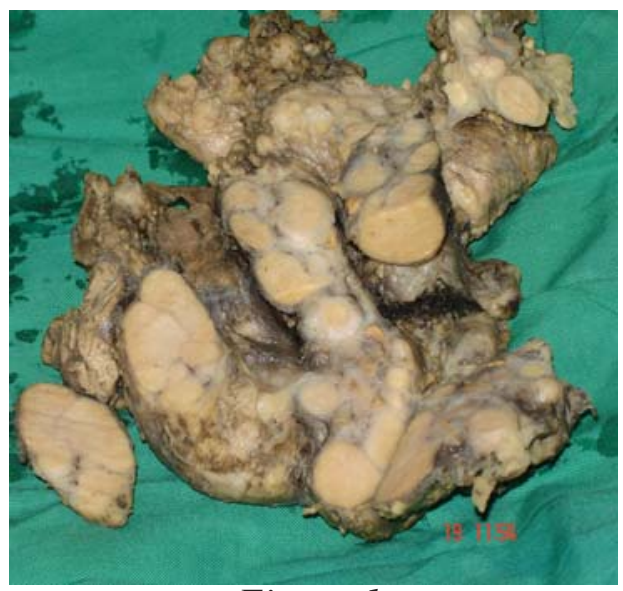

Figure 1 
Microscopic examination was characteristic with capsular fibrosis, partial effacement of nodal architecture and dilated medullary sinuses packed with histiocytes (Fig 2). The intersinusal tissue contained numerous mature plasma cells with many bearing Russell's bodies. A tentative diagnosis of Nodal Rosai Dorfman disease was made.

Ziehl Neelson, Wade Fite and DPAS stains were negative. Immunohistochemical stains for S100 proteins was positive confirming our diagnosis of Nodal Rosai Dorfman disease.

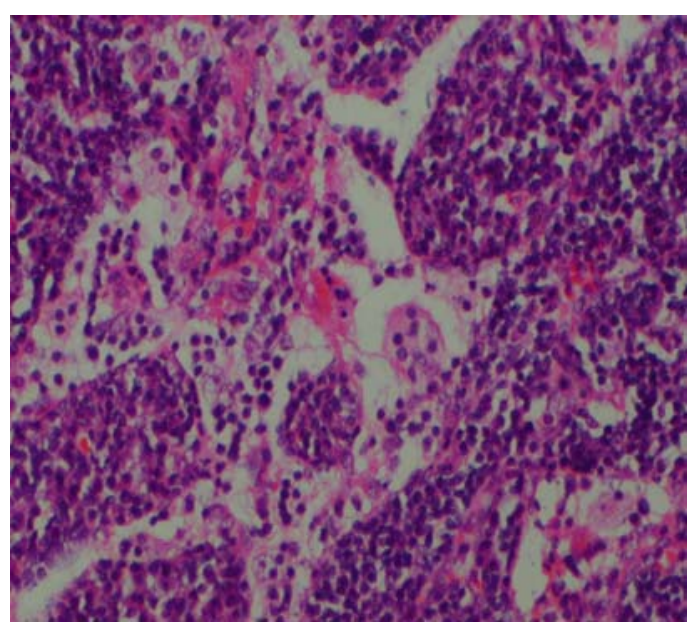

Figure 2

\section{Discussion}

RDD most commonly occurs in children and young adults, although individuals of almost any age can be affected [2]. The majority of cases present with painless cervical lymphadenoapthy, often bilateral and massive. Although cervical node involvement is by far the commonest, other lymph node groups can be affected with or without cervical node involvement.
Microscopically, there is an effacement of nodal architecture with prominent dilatation of the sinuses. The sinuses are filled with the characteristic macrophages with round to oval vesicular nuclei and abundant vacuolated cytoplasm. Lymphocytes, some times plasma cells and rarely red cells are seen within the cytoplasm of these histiocytes a feature termed emperipolesis. This is a constant feature in RDD and is of great diagnostic significance.

The aetiopathogenesis of RDD remains uncertain [3]. RDD is usually a benign self limiting disease that resolves without any treatment in approx. 20\% of cases, although it could take several years. A very small proportion of patients develop widespread disease with a few reported deaths attributed to RDD. Currently there is no ideal treatment available. Various treatment modalities including surgery, chemotherapy, radiotherapy steroids, alpha interferon and acyclovir have been used in RDD with variable success [4].

\section{References}

1. Rosai J, Dorfman RF. Sinus Histiocytosis with massive lymphadenopathy: a newly recognised benign clinicopathological entity. Archive Pathology 1969.

2. Fouca F, Rosai J, Dorman R. Sinus Histiocytosis with massive Lymphadenopathy (Rosai Dorfman Disease): Review of the Entity. Semin Diagnostic Pathology 1990; 7: 19-73.

3. Rosai and Ackerman's Surgical Pathology, $9^{\text {th }}$ edition 2004: 1911-30.

4. Komp DM. The treatment of sinus histiocytosis with massive lymphadenoapthy (RDD). Semin Diagnostic Pathology 1990; 7: 83-6. 